\title{
AN ANALYTICAL APPROACH TO THE DESCRIPTION OF NONADIABATIC CELLULAR FLAMES NEAR EXTINCTION*
}

\author{
L. SINAY $¥ \ddagger$ AND F. A. WILLIAMS
}

\begin{abstract}
Parametric expansions in power series are employed to obtain steady, spatially periodic solutions 10 a pair of evolution equations for flame shape and flame temperature of premixed flames with heat loss in a thermal-diffusional approximation. The approach results in a computational method that is as accurate as earlier numerical integrations hut much quicker. Consequently, improved accuracy is attained in predicting the maximum rate of heat loss for flame propagation, the wavelength at this miaximum, burning velocities, and flame shapes. The results have a bearing on the behaviors of flames near the extinction limit.
\end{abstract}

Key words. cellular flames, bifurcation, parametric expansions

AMS(MOS) subject classifications. $80 \mathrm{A3} 2$

1. Introduction. Joulin and Sivashinsky [1] derived a system of two partial differential equations for describing the dynamics of premixed flame fronts near the condition of extinction by heat loss under the restriction that the planar flame is unstable to cellular perturbations. Joulin [2] integrated steady-state versions of these equations numerically to demonstrate that there are solutions for certain cellular flames that propagate steadily with rates of heat loss greater than the limiting value for extinction of the planar flame. The absence of a complete analysis of the problem prompted the present investigation of steady solutions to the Joulin-Sivashinsky equations. The objective is to improve the accuracy and speed of obtaining solutions and to explore how cellular flame phenomena extend the propagation limit.

The flame-front equations are derived [1] under the assumption that the small parameter $\varepsilon=(1-L e)\left(T_{b}-T_{u}\right) T_{a} /\left(6 T_{h}^{2}\right)$ is positive, where Le (the ratio of thermal-to. mass diffusivity) is the Lewis number of the limiting reactant, $T_{h}$ the adiabatic flame temperature, $T_{u}$ the temperature of the unburnt gas, and $T_{u}$ the activation temperature for the reaction rate. Near the limit of extinction by Newtonian heat loss, the assumption that $\varepsilon>0$ places the flame in the cellular regime rather than in the stable or pulsating regime. The description applies when the scaled heat-loss deviation parameter $\nu=$ $(l e-1) /\left(2 \varepsilon^{2}\right)$ is of order unity, where $l$ is the heat-loss parameter [3] that achieves the critical value $e^{-1}$ at extinction of the planar flame with Lewis number unity. Additional approximations underlying the equations are that the thermal-diffusional approxima. tion of constant gas density applies and that the Zel'dovich number $\left(T_{b}-T_{w}\right) T_{w i} / T_{h}^{2}$ is large (a highly temperature-sensitive reaction rate), so that Le must be very near unity.

The thermal diffusivity $D$ and the burning velocity $U$ of the planar flame with $L e=1$ at extinction provide natural length and timescales for flame response. For the planar flame near the extinction point, the propagation velocity is $U(1+\mu \varepsilon)$, where the nondimensional increment of propagation velocity $\mu$ is given by the parabola $\mu^{2}+\nu=0$. The nondimensional space $\zeta$ and time $\tau$ are measured in units of $D /\left(r^{1 / 2} U\right)$ and $D /\left(\varepsilon U^{2}\right)$, respectively, so that attention is focused on slow evolution. If the front position (in units $D / U$ ) in a frame moving loward the fresh mixture with velocity $U$

- Received by the editors July 9, 1990; accepted for publication (in revised form) May 15, 1991. This research was supported in part by National Science Foundation grant CTS-8918527.

$\uparrow$ Department of Applied Mechanics and Engineering Sciences, University of California at San Diego. La Jolla, California 92093.0310.

¥ This author is on leave from the Space Research Institute, Instituto de Pesquisas Espaciais, Bravil. under the sponsorship of the Brazilian National Research Council-CNPq, project number 202202/84.4

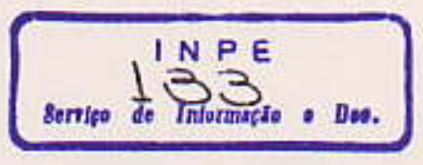


is denoted by $\phi$, and a nondimensional flame-temperature decrement is defined as $\psi=\left[\left(T_{b}-T\right) T_{a} / T_{b}^{2}-1\right] /(2 \varepsilon)$, where $T(\tau, \zeta)$ is the local, instantaneous, reaction-zone temperature, then the Joulin-Sivashinsky evolution equations become [1]

$$
\begin{array}{ll}
\phi_{\tau}+\frac{1}{2}|\nabla \phi|^{2}=\Delta \phi+\psi & \text { on } \Omega \times \mathbb{R}, \\
\psi_{\tau}+\nabla \psi \cdot \nabla \phi=\Delta \psi-\Delta \phi+\frac{1}{3}\left(\psi^{2}+\nu\right) & \text { on } \Omega \times \mathbb{R}, \\
\partial \phi / \partial n=\partial \psi / \partial n=0 & \text { on } \partial \Omega,
\end{array}
$$

where $\Omega$ is a region in $\mathbb{R}$ or $\mathbb{R}^{2}, \partial \Omega$ is its boundary, and $\partial / \partial n$ means that the derivative in the direction of the normal.

Joulin [2] determined numerical solutions to (1.1) in one space dimension for steady-state flames propagating at a constant velocity increment $\mu$. He employed a shooting method and found that many different cellular patterns can exist at the same heat-loss parameter $\nu$, each having its own $\mu$ and maximum heat-loss parameter $\nu_{m}$. The purpose of the present paper is to give an analytical method for obtaining such solutions.

2. Smooth, steady, periodic solutions. In this section we address the onedimensional form of system (1.1) for a constant propagation velocity and consider solutions that are periodic in $\zeta$. If we let $\phi=-\mu \tau+f, \psi=-\mu+g$, then, after substitution into the one-dimensional form of (1.1), we have

$$
\begin{array}{ll}
f_{7}+\frac{1}{2} f_{\zeta}^{2}=f_{G}+g & \text { on } \Omega \times \mathbb{R}, \\
g_{\tau}+f_{6} g_{\zeta}=g_{G}-f_{G \zeta}+\frac{1}{3}\left(\mu^{2}-2 \mu g+g^{2}+\nu\right) & \text { on } \Omega \times \mathbb{R}, \\
f_{\zeta}=g_{6}=0 & \text { on } \partial \Omega .
\end{array}
$$

Let $x_{1}=f_{6}, x_{2}=g_{1}, x_{3}=g_{6}$, and $\nu=-\mu^{2}+\sigma$, and then assume that $f$ and $g$ are independent of $\tau$. Introduce $z=k \zeta$ as a new variable, where $k=2 \pi / L$, in which $L$ is the selected period, and let a prime denote the derivative with respect to $z$. Then we obtain from (2.1)

$$
\begin{array}{ll}
k x_{1}^{\prime}=\frac{1}{2} x_{1}^{2}-x_{2}, & \\
k x_{2}^{\prime}=x_{3}, & \text { on }[0,2 \pi] \\
k x_{3}^{\prime}=\frac{1}{2} x_{1}^{2}-x_{2}-x_{1} x_{3}-\frac{1}{3}\left(x_{2}^{2}-2 \mu x_{2}+\sigma\right), &
\end{array}
$$

and

$$
x_{j}(0)=x_{j}(2 \pi)=0, \quad j=1,3 .
$$

It seems of interest to remark that standard methods prove the existence of smooth periodic solutions to $(2.2),(2.3)$. In summary, if the operator $F$ is defined by

$$
F[X, \lambda]=k X^{\prime}-R(X),
$$

where $X=\left(x_{1}, x_{2}, x_{3}\right)^{\prime}, \lambda=(\mu, \sigma) \in \mathbb{R}^{2}$, and

$$
R(X)=\left(\frac{1}{2} x_{1}^{2}-x_{2}, x_{3}, \frac{1}{2} x_{1}^{2}-x_{2}+x_{1} x_{3}-\frac{1}{3}\left(x_{2}^{2}-2 \mu x_{2}+\sigma\right)\right)^{\prime},
$$

then $F[0, \lambda]=0$ when $\lambda=(\mu, 0)$ for all $\mu$, and the variational equation around this solution has the nontrivial solution

$$
\Phi=\left(-a k \sin z, a k^{2} \cos z,-a k^{3} \sin z\right)^{\prime}, \quad a=1 / \sqrt{1+k^{4}}
$$

when $\mu=\mu_{0}$, where $\mu_{0}=3\left(1-k^{2}\right) / 2$. If Frechèt derivatives are identified by subscripts and the superscript 0 denotes their evaluation at $\mu=\mu_{0}, \sigma=\sigma_{6}$, where $\sigma_{6}=0$, then the operator $F_{x}^{\prime \prime}+F_{x \lambda}^{0} \Phi$ has the properties needed $[4]$ to prove that the equation $F[x, \lambda]=0$ 
has a $2 \pi$-periodic solution, which bifurcates from $\left(X_{0}, \mu_{0}, \sigma_{0}\right)=\left(0,3\left(1-k^{2}\right) / 2,0\right)$ and which can be expanded in the power series

$$
X=\sum_{n=1}^{\infty} X_{n} \delta^{n}, \quad \mu=\sum_{n=0}^{\infty} \mu_{n} \delta^{n}, \quad \sigma=\sum_{n=1}^{\infty} \sigma_{n} \delta^{n},
$$

where

$$
\delta^{2}=\frac{1}{\pi} \int_{0}^{2 \pi}\langle X ; X\rangle d z
$$

in which $\langle;\rangle$ is the dot product in $\mathbb{R}^{3}$.

3. The mathematical algorithm. Bifurcation of periodic solutions of nonlinear ordinary differential equations is perhaps one of the most frequently sought types of bifurcation. Often these solutions are determined in an approximate way that involves cumbersome calculations by means of a perturbation method. In this section we present an algorithm that is a combination of power series and Fourier series and that allows the determination of the periodic solutions of quadratic equations to any desired degree of approximation. The method is based on the ideas presented by Levy and Keller [5]. More recent applications of similar schemes can be found, for example, in [6]-[10]. The fact that the equation is quadratic is not a fundamental restriction since the principle of the method can be utilized for any polynomial nonlinearity. However, the complexity of the calculation increases with increasing degree of the polynomial.

To avoid unnecessary subscripts, we return to the $f, g$ notation. Using $(2.5)$ in (2.1) and recalling that $\Omega$ was transformed into the interval $[0,2 \pi]$, we obtain

$$
\begin{gathered}
k^{2} \frac{\partial^{2} f_{n}}{\partial z^{2}}+g_{n}= \\
k^{2} \frac{k^{2}}{2} \sum_{j=1}^{n-1} \frac{\partial f_{j}}{\partial z} \frac{\partial f_{n-1}}{\partial z}, \\
+\frac{\partial^{2} g_{n}}{\partial z^{2}}+\frac{2}{3} \mu_{0} g_{n}=-\frac{2}{3} \sum_{j=1}^{n-1} \mu_{j} g_{n-1}-k^{2} \sum_{j=1}^{n-1} \frac{\partial f_{j}}{\partial z} \frac{\partial g_{n-j}}{\partial z}+\frac{1}{3} \sigma_{n} ; \\
\frac{\partial f_{n}}{\partial z}=\frac{\partial g_{n}}{\partial z}=0 \quad \text { at } z=0,2 \pi ; \\
\frac{1}{\pi} \int_{0}^{2 \pi} f_{n} d z=0, \\
\frac{a}{\pi} \int_{0}^{2 \pi}\left(f_{n}(z)+k^{2} g_{n}(z)\right) \cos z d z=\delta_{n 1} \quad(\delta \text { Kronecker's delta). }
\end{gathered}
$$

Here and in what follows, sums with upper summation limit smaller than the lower one must be considered nil.

If the fundamental period of the solution of ( $3.1 \mathrm{a})$ is chosen to be $2 \pi$ when $n=1$, then $f_{n}(z)$ and $g_{n}(z)$ can be shown to be finite sums of the form

$$
f_{n}(z)=\sum_{p=0}^{n} \hat{f}_{n}(p) \cos p z, \quad g_{n}(z)=\sum_{z=0}^{n} \hat{g}_{n}(q) \cos q z .
$$

Equation (3.2) can be derived by induction on $n$; when $n=1$ all the sums on the right-hand sides of (3.1a) are nil, and then

$$
f_{1}(z)=a \cos z, \quad g_{1}(z)=a k^{2} \cos z,
$$


so (3.2) is true in this case. If it is assumed that (3.2) is also true up to $n-1$, then since the subscripts on the right-hand side of (3.1a) range from 1 to $n-1$, it is possible to apply the inductive hypothesis. In doing so, we must observe that the terms are either linear, involving $g_{n-1}$, and therefore given by $(3.2)$ with $q \leqq n-j$, or products with indices $j$ and $n-j_{1} j$ running from 1 to $n-1$. Using (3.2) for them, we obtain, for example,

$$
\frac{\partial f_{j}}{\partial z} \frac{\partial f_{n-j}}{\partial z}=\left(\sum_{p=1}^{j} p \hat{f}_{j}(p) \sin p z\right)\left(\sum_{q=1}^{n-j} q \hat{f}_{n-j}(q) \sin q z\right) .
$$

Expanding the products, we get terms of the form

$$
\sin p z \sin q z=\frac{1}{2}[\cos (p-q) z-\cos (p+q) z]
$$

with $1 \leqq p \leqq j, 1 \leqq q \leqq n-j$; consequently, $0<|p-q| \leqq n$ and $p+q \leqq n$. Since the homogeneous equation has a nontrivial solution, to have periodic solutions, secular terms must be removed from (3.1a) by an adequate choice of $\sigma_{n}$ and $\mu_{n-1}$, a process that does not introduce new harmonics. Finally, because $(3.1 \mathrm{a})$ is linear, integration of the equation is achieved by superposition of particular solutions, all of them harmonics of the same type as the corresponding right-hand side of (3.1a).

We can now substitute for $f_{i}(z)$ and $g_{i}(z)(1 \leqq i \leqq n)$ in $(3.1 \mathrm{a})-(3.1 \mathrm{c})$ the forms (3.2) and, since these are finite sums, perform term-by-term differentiation. Collecting like terms, we obtain

$$
\begin{gathered}
\hat{g}_{n}(0)=r_{01}, \\
\frac{2}{3} \mu_{0} \hat{g}_{n}(0)=\frac{1}{3} \sigma_{n}+r_{02}, \\
\mathbf{M}[p] \mathbf{y}_{n}[p]=\mathbf{A}[p] \mu_{n-1}+\mathbf{R}_{n}[p], \\
\hat{f}_{n}(1)+k^{2} \hat{g}_{n}(1)=\delta_{n 1}, \\
\hat{f}_{n}(0)=0,
\end{gathered}
$$

where

$$
\mathbf{M}[p]=\left[\begin{array}{cc}
-k^{2} p^{2} & 1 \\
k^{2} p^{2} & 1+\left(p^{2}-1\right) k^{2}
\end{array}\right], \quad \mathbf{y}[p]=\left[\begin{array}{c}
\hat{f}_{n}(p) \\
\hat{g}_{n}(p)
\end{array}\right], \quad \mathbf{A}[p]=\left[\begin{array}{c}
0 \\
-\hat{g}_{1}(p)
\end{array}\right],
$$

$$
\begin{gathered}
r_{01}=\frac{k^{2}}{4} \sum_{j=1}^{n-1} l^{2} \hat{f}_{j}(l) \hat{f}_{n-j}(l), \\
r_{02}=\sum_{j=1}^{n-1}\left\{-\frac{2}{3} \mu_{j} \hat{g}_{n-j}(0)-\frac{k^{2}}{2} \sum_{i=1}^{\infty} l^{2} \hat{f}_{j}(l) \hat{g}_{n-j}(l)\right. \\
\left.+\frac{1}{6}\left[\hat{g}_{j}(0) \hat{g}_{n-j}(0)+\sum_{l=0}^{\infty} \hat{g}_{j}(l) \hat{g}_{n-j}(l)\right]\right\},
\end{gathered}
$$

and $R_{n}[p]$ are the two-dimensional vectors $\left(R_{n_{1}}[p], R_{n_{2}}[p]\right)^{\prime}$ defined by

$$
\begin{aligned}
R_{n 1}[p]= & -\frac{k^{2}}{4} \sum_{j=1}^{n-1}\left\{\sum_{i=1}^{p-1} I(p-l) \hat{f}_{j}(l) \hat{f}_{n-j}(p-l)\right. \\
& \left.-\sum_{i=1}^{\infty} I(p+l)\left[\hat{f}_{j}(l) \hat{f}_{n-j}(p+l)+\hat{f}_{j}(p+l) \hat{f}_{n-j}(l)\right]\right\},
\end{aligned}
$$




$$
\begin{aligned}
R_{n 2}[p]= & -\frac{1}{3} \sum_{j=1}^{n-2} \mu_{j} \hat{g}_{n-j}(p) \\
& +\frac{1}{2} \sum_{j=1}^{n-1}\left\{k ^ { 2 } \left[\sum_{i=1}^{p-1} I(p-l) \hat{f}_{j}(l) \hat{g}_{n-j}(p-l)\right.\right. \\
& \left.-\sum_{i=1}^{\infty} l(p+l)\left(\hat{f}_{j}(l) \hat{g}_{n-j}(p+l)+\hat{f}_{j}(p+l) \hat{g}_{n-j}(l)\right)\right] \\
& \left.+\frac{1}{3}\left[\sum_{i=0}^{p} \hat{g}_{j}(l) \hat{g}_{n-j}(p-l)+\sum_{l=0}^{\infty}\left(\hat{g}_{j}(p+l) \hat{g}_{n-j}(l)+\hat{g}_{j}(l) \hat{g}_{n-j}(p+l)\right)\right]\right\} .
\end{aligned}
$$

To simplify the notation, we use $\infty$ as the upper summation limit in some of the terms. However, we must recall that they are actually finite sums since, by $(3.2), \hat{f}_{n}(p)=\hat{g}_{n}(p)=$ 0 if $p>n$. We have thus transformed the nonlinear boundary-value problem $(2.2)$ into a sequence of linear, algebraic equations that can be solved recursively. Observe that $\operatorname{det} \mathbf{M}[p]=\left(p^{2}-1\right) p^{2} k^{4} ;$ thus $M[p]$ is singular only when $p=1$.

Assuming that $(3.3 \mathrm{a})-(3.5 \mathrm{~b})$ have been solved for $1 \leqq j \leqq n-1$ for all $p$, we start solving the systems for $j=n$ choosing first the case where $p=1$. Since $M[1]$ is singular, (3.4) has a solution if and only if the right-hand side is orthogonal to the kernel of the adjoint matrix $\mathbf{M}[1]^{\prime}$, which is spanned by $V=(1,-1)^{\prime}$; hence

$$
\mu_{n-1}=3 \frac{\left\langle V ; \mathbf{R}_{n}[1]\right\rangle}{2 a k^{2}} \text {. }
$$

Condition (3.5a) then makes the solution of (3.4) unique. Equation (3.3a) defines $\hat{g}_{n}(0)$, and using this in $(3.3 \mathrm{~b})$ we obtain $\sigma_{n} ; \hat{f}_{n}(0)$ is already defined by $(3.5 \mathrm{~b})$. Finally, $\mathbf{y}_{n}[p], 2 \leqq p \leqq n$ can be calculated from (3.4) using (3.7) for $\mu_{n-1}$.

The procedure just outlined is then a sequence of recursive solutions of $2 \times 2$ algebraic systems, which can be simplified further by observing that $(3.3 \mathrm{a})-(3.5 \mathrm{~h})$ yield and (b) $\mu_{n}=\sigma_{n}=0$ if $n$ is odd. This may be dementies (one is even and the other odd) that (a) is trivially true for $n=1$ because demonstrated by induction by first noting $\hat{g}_{1}(1)$. For (b), equations (3.3a) (3.6a) the only nonzero coefficients are $\hat{f}_{1}(1)$ and in (3.6c), (3.6d) with $n=2$ (a) substitution (a) and (b) apply up to $n-1$ ives $R_{21}[1]=R_{22}[1]=0$; hence $\mu_{1}=0$. If it is assumed that if $n$ is even and $\sigma_{2 j+1}=0, \mu_{2,+}=0,1 \leqq 2 j+1 \leqq n-1, \mu_{2 i+1}=0,1 \leqq 2 j+1 \leqq n-2$ case where $n$. Let us consider the case where $n$ is even, then, when $p=1$, the products $\hat{f}_{,}(l+l) \hat{f}_{n-1}(l)$ in $(3.6 \mathrm{c})$ are zero different parities, and then $\hat{f}_{n-1}(l)=0$. The same reach case $\hat{f}_{1}(l+1)=0$, or they have of $\hat{f}$ 's and $\hat{g}$ 's. When $j$ is even, $\mu$. The same reasoning applies to the other products $\mu_{j}=0$ for $j \leqq n-2$. Hence $\mu_{j} \hat{g}_{n-j}(1)=0$ because $\hat{g}_{n-j}(1)$ is even; when $j$ is odd, $-2 \mu_{n-1} \hat{g}_{1}(1)$, respectively, and therefore $\mu_{n-1}=0$. Then $\hat{f}_{(1)}$ are reduced to zero and token, (3.6c), (3.6d) yield zero right-hand sid $\mu_{n-1}=0$. Then $\hat{f}_{n}(1)=\hat{g}_{n}(1)=0$. By the same 1 above); thus $\hat{f}_{n}(p)=\hat{g}_{n}(p)=0$. If $n$ is odd, the reasoning applies, mutatis mutandi, to show that $\sigma_{n}$ and $\hat{g}_{n}(0)$ are zero from (3.6a), (3.6b), and $\hat{f}_{n}(p)=\hat{g}_{n}(p)=0$ if $p$ is even.

4. Singular solutions. We have seen in $\$ 3$ that the determinant of the matrix of system (3.4) is $k^{4} p^{2}\left(1-p^{2}\right)$, which, since the system is defined only for $p>0$, vanishes only when $p=1$ if $k \neq 0$, or identically if $k=0$. Let us consider now the case in which $k$ is very small and use singular perturbation techniques to determine the asymptotic behavior of the static solutions of (2.1). Substituting $f, g$ and $h=\nu+\mu^{2}$ into the equations 
by, respectively, $\sum_{n=0}^{\infty} f_{n} k^{n}, \sum_{n=0}^{\infty} g_{n} k^{n}$, and $\sum_{n=0}^{\infty} h_{n} k^{n}$, and collecting like powers of $k$, we obtain $g_{0}=g_{1}=0, h_{0}=h_{1}=0$, and

$$
(3 \mu-1) f_{0}^{\prime \prime}-\frac{1}{3} \mu\left(f_{0}^{\prime}\right)^{2}=-\frac{1}{3} h_{2} .
$$

It is easy to prove by induction that if $\mu=\frac{3}{2}$ then $f_{n}=$ const, $g_{n}=0, h_{n}=0$ for all $n$; i.e., the trivial solution of $(2.1)$ is obtained. If $\mu \neq \frac{3}{2}$, then

$$
\begin{aligned}
& f_{0}(z)= \frac{2}{\mu}\left(\frac{3}{2}-\mu\right) \ln \left|\cos \left(\frac{z}{2}\right)\right|, \\
& f_{1}(z)=0,=\frac{1}{2\left(\mu-\frac{3}{2}\right) \mu^{4}}\left\{\left(\mu^{3}-\left(\frac{3}{2}\right)^{3}\right) \ln \left|\cos \left(\frac{z}{2}\right)\right|-\frac{3}{4}\left(\frac{3}{2}-\mu\right)\right. \\
&\left.f_{2}(z)=\left(\mu+\frac{3}{a^{+}-2}\right)\left(\mu+\frac{3}{a^{-}-2}\right) \frac{1}{\cos ^{2}(z / 2)}\right\}, \\
& g_{2}(z)=-\frac{1}{2}\left(\frac{\mu-\frac{3}{2}}{\mu}\right)^{2}+\left[\frac{3\left(\frac{3}{2}-\mu\right)}{4 \mu^{2}}\right] \frac{1}{\cos ^{2}(z / 2)}, \\
& h_{2}=-\frac{1}{\mu}\left(\frac{3}{2}-\mu\right)^{2}, \\
& h_{4}=\frac{1}{32 \mu^{4}}\left(\frac{3}{2}-\mu\right)\left(8 \mu^{3}+12 \mu^{2}+126 \mu+81\right),
\end{aligned}
$$

where $a^{ \pm}=7 \pm \sqrt{13}$.

Although no claim can be made about the convergence of the series, (4.1) shows that the asymptotic behavior of $f(z)$ is determined by $\ln |\cos (z / 2)|$. In particular, when $\mu=\mu^{ \pm}=-3 /\left(a^{ \pm}-2\right)$ and $k \rightarrow 0$,

$$
f(z) \sim f^{*}(z)=-k a^{2} \ln |\cos (z / 2)| .
$$

In fact, $F(\zeta, k)=-a^{ \pm} \ln |\cos (k \zeta / 2)|$ is an exact solution of (2.1) obtained previously [1], [2], provided that

$$
\begin{aligned}
g(\zeta) & =-\frac{\left(a^{ \pm} k\right)^{2}}{8}+\frac{\left(a^{ \pm}-z\right) a^{5} k^{2}}{8 \cos ^{2}(k \zeta / 2)}, \\
\mu & =-\frac{3}{a^{x}-2}-\left(a^{ \pm}-3\right) k^{2},
\end{aligned}
$$

and

$$
\nu=-\left[\frac{3}{a^{ \pm}-2}+\left(a^{ \pm}-3\right) k^{2}\right]^{2}-\frac{\left(a^{ \pm} k\right)^{2}}{64}\left(\left(a^{ \pm} k\right)^{2}+16 \mu\right),
$$

and (4.2) approaches this solution as $k$ approaches zero, so that, in a sense, it is a member of the class derived here. It is "less singular" than the other solutions determined by (4.1) in that the divergence as $k \zeta \rightarrow \pm \pi$ is slower, and therefore it is likely to be preferred physically. Since the cusps at the singularities extend to infinity, they could occur in the interior of a domain only if they are truncated by failure of the scaling and intrusion of additional physical phenomena, as suggested below. Therefore they seem more likely to occur at the walls for flames propagating in tubes or channels. For example, upwardly propagating flames in tubes exhibit long skirts pointing downward when the experimental conditions are near the limits of flammability 
[3]. The singular solutions may bear relevance to such flames, although further investi. gation of effects of buoyancy and of flow would be needed to ascertain whether this is true.

It is clear from (4.3) and (4.4) that as $k \rightarrow 0, \mu \rightarrow \mu^{2}$ and $\nu \rightarrow-\left(\mu^{2}\right)^{2}$. We have thus shown that, in the neighborhood of the parabola $\nu+\mu^{2}=0,(2.1)$ possesses the smooth periodic solutions determined in $\$ 3$ and two singular periodic solutions with asymptotic expansions given by $(4.1)$.

5. Periodic solutions and their properties. Trajectories in the $\nu, \mu$ plane, predicted by (4.3) and (4.4), are shown in Fig. 1. They exhibit the same maximum value of $\nu$,

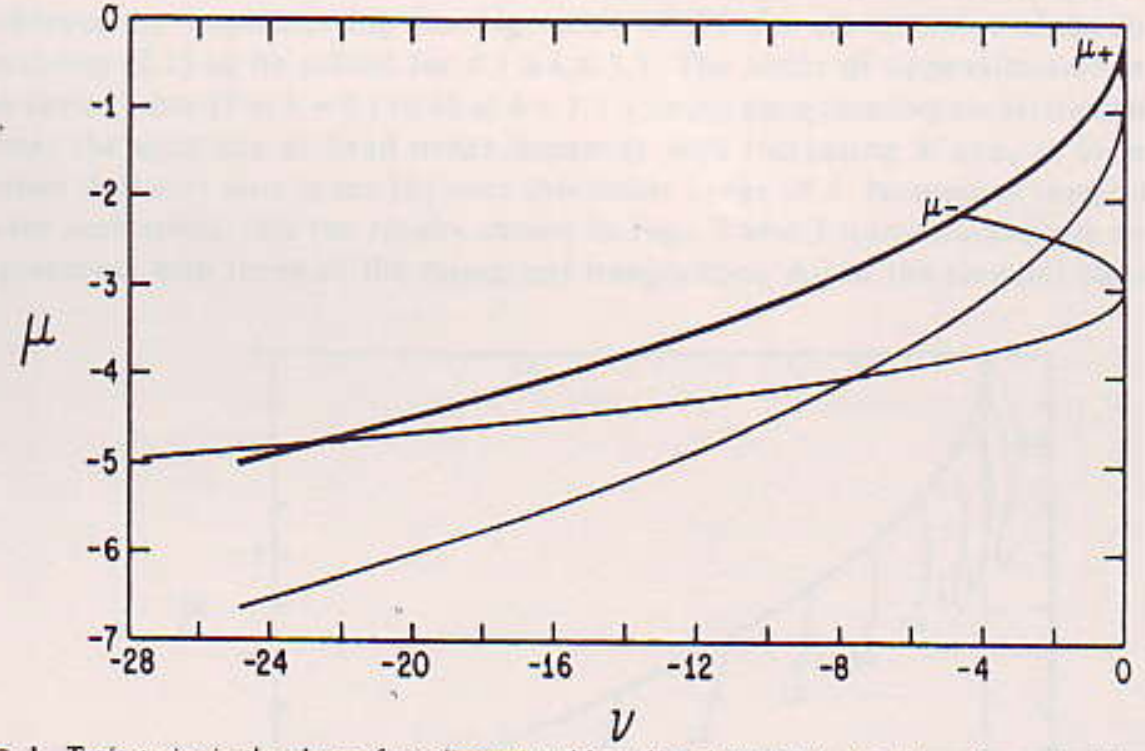

Fic. 1. Trajectories in the plane of nondimensional heat-loss deviation $(\nu)$ and nondimensional burning. velocity deviation $(\mu)$ of the two sets of singular solutions (light lines), bifurcating from the trajectory of the planar-front solutions (heavy line).

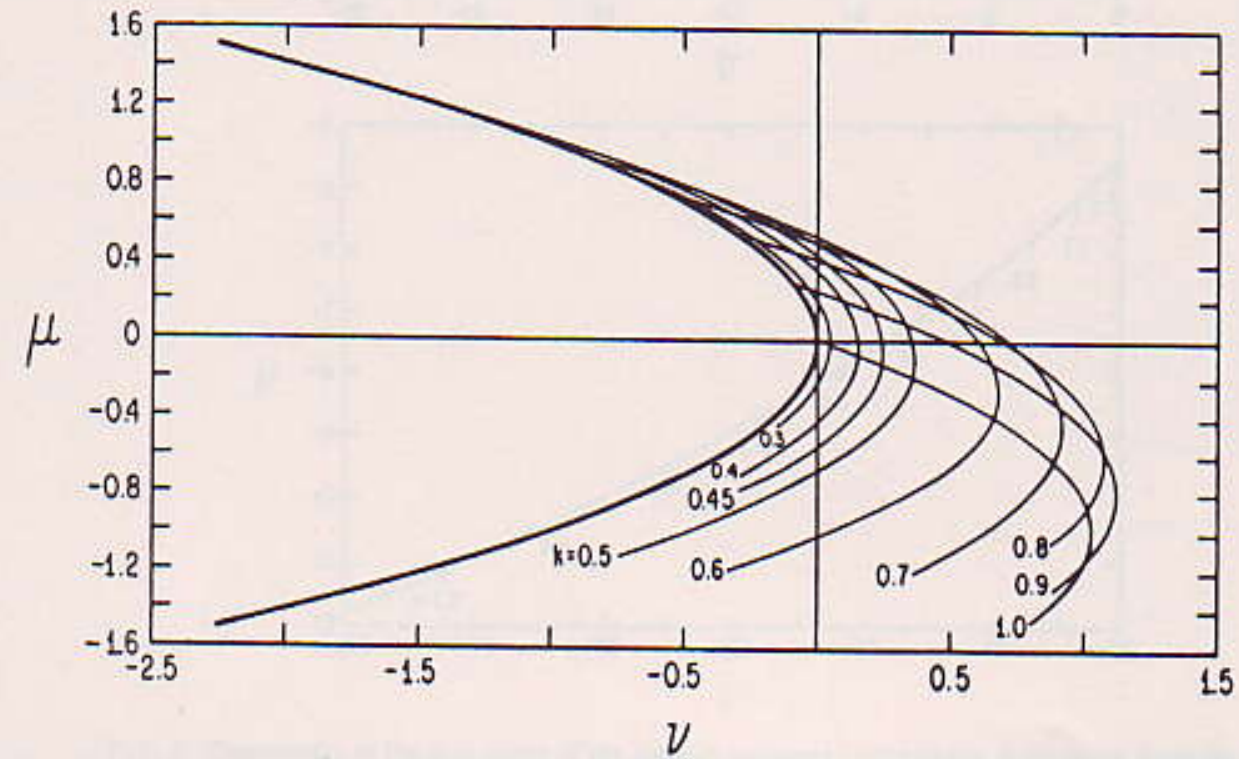

FIC. 2. Trajectories in the $\nu, \mu$ plane of the smooth solutions (light lines), bifurcating from the trajectory of the planar-front solutions (heavy line) for $k \leqq 1$. 
zero, at, respectively, $k^{2}=\left(16-5 a^{ \pm}\right) /\left(322-95 a^{ \pm}\right)=(0.05401,2.05710)$, the first of which, at least, should be well within the range of the fourth-order expansion given in (4.1). This shows that the extinction limit (the maximum $\nu$ ) of these curved flames is the same as that of the planar flame. As indicated above, these singular solutions can be of interest not only for flame propagation in channels with walls separated by a distance $\left[D /\left(\varepsilon^{1 / 2} U\right)\right](2 \pi / k)$, but possibly also in the open, where cusp closure or reactant leakage through the cusps may occur in a range of solutions beyond the scaling considered here.

Corresponding trajectories for the smooth solutions are shown in Figs. 2 and 3 , which extend results given earlier [2] to wider ranges of $\nu$ and $k$. The extension was achieved by implementing the algorithm of $\$ 3$ for numerical evaluation, thereby enabling (2.1) to be solved for $0.1 \leqq k \leqq 3.5$. The order of approximation in $\delta$ could be varied from 17 at $k=0.1$ to 45 at $k=3.5$ without encountering excessive computation time: the accuracy at fixed order improves with increasing $k$ and, at order 17 , was hetter than one part in ten [6] over this entire range of $k$. Numerical integrations also were performed, and the results shown in figs. 2 and 3 were found to be in excellent agreement with those of the numerical integrations. All of the singular solutions that
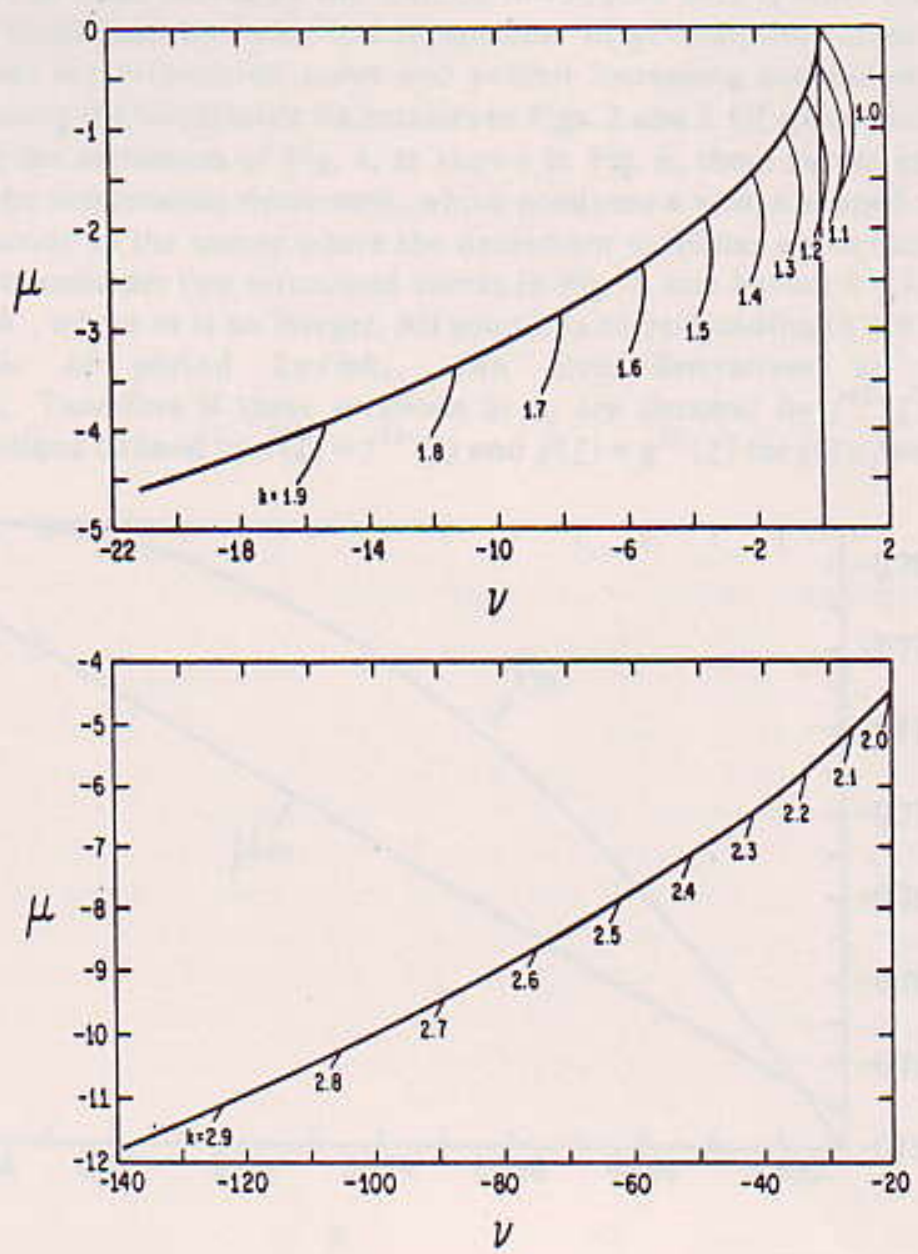

Fic. 3. Trajectories in the $\nu_{1} \mu$ plane of the smooth solutions (light lines), bifurcating from the trajectory of the planar.front solutions (heavy line) for (a) $1 \leq k \leq 1.9$ and (b) $2 \leqq k \leq 2.9$. 
have been found bifurcate from the planar solution in the region contained in Fig. 3a, as may be seen by comparing Figs. 1 and $3 a$.

One of the most remarkable findings of Joulin [2] was that certain nonplanar flames can propagate with heat-loss rates greater than the maximum that extinguishes the planar flame. This may be seen in Fig. 2, which shows that there are no planar solutions for $\nu>0$, but some bifurcated solutions extend into the range $\nu>1$. It was found [2] that the maximum value of $\nu$ for which nonplanar solutions could be obtained was $\nu \approx 1.13$, which occurred with $k \approx 0.9$. By numerical integration using Richardson's extrapolation method, we found that $\nu=1.1285$ for the extremum, at which value the burning-velocity deviation was $\mu=-0.7950$. Results of the perturbation scheme for the maximum value of $\nu$, denoted by $\nu_{m}$, and for the value of $\mu$ at this maximum, denoted by $\mu_{m}$, as functions of $k$ are shown in Fig. 4. The perturbation results indicate that the extremum occurs at $k \approx 0.888$ and has $\nu_{m}=1.1294$ and $\mu_{m}=-0.7686$. Thus at the extremum the results of the perturbation method agree with those of the numerical integration to within three or four significant figures.

Representative flame shapes $f(z)$ and temperature decrements along the reaction sheet $g(z)$, as obtained from the perturbation solution, are shown in Fig. 5. The numerical generation of these curves by the method introduced here is more than an order of magnitude faster than by numerical integration. In general, the curves have unimodal shapes near the bifurcation point and exhibit increasing oscillation with increasing distance along the bifurcation trajectories in Figs. 2 and 3. Of special interest are $f(z)$ and $g(z)$ at the extremum of Fig. 4, as shown in Fig. 6; these curves exhibit rapid oscillation in the temperature decrement, which produces a wedge-shaped flame with a nearly flat portion in the center where the decrement oscillates about zero.

It is of interest to consider two bifurcated curves in Fig. 2, one having $k=k_{1}$ and the other $k=k_{2}=m k_{1}$, where $m$ is an integer. All solutions corresponding to the latter curve are periodic of period $2 \pi / m k_{1}$, with zero derivatives at $\zeta=0$, $2 \pi / m k_{1}, \cdots, 2 \pi / k_{1}$. Therefore if these solutions at $k_{2}$ are denoted by $f^{(2)}(\zeta)$ and $g^{(2)}(\zeta)$, then the functions defined by $\tilde{f}(\zeta)=f^{(2)}(\zeta)$ and $\tilde{g}(\zeta)=g^{(2)}(\zeta)$ for $j\left(2 \pi / m k_{1}\right) \leqq$

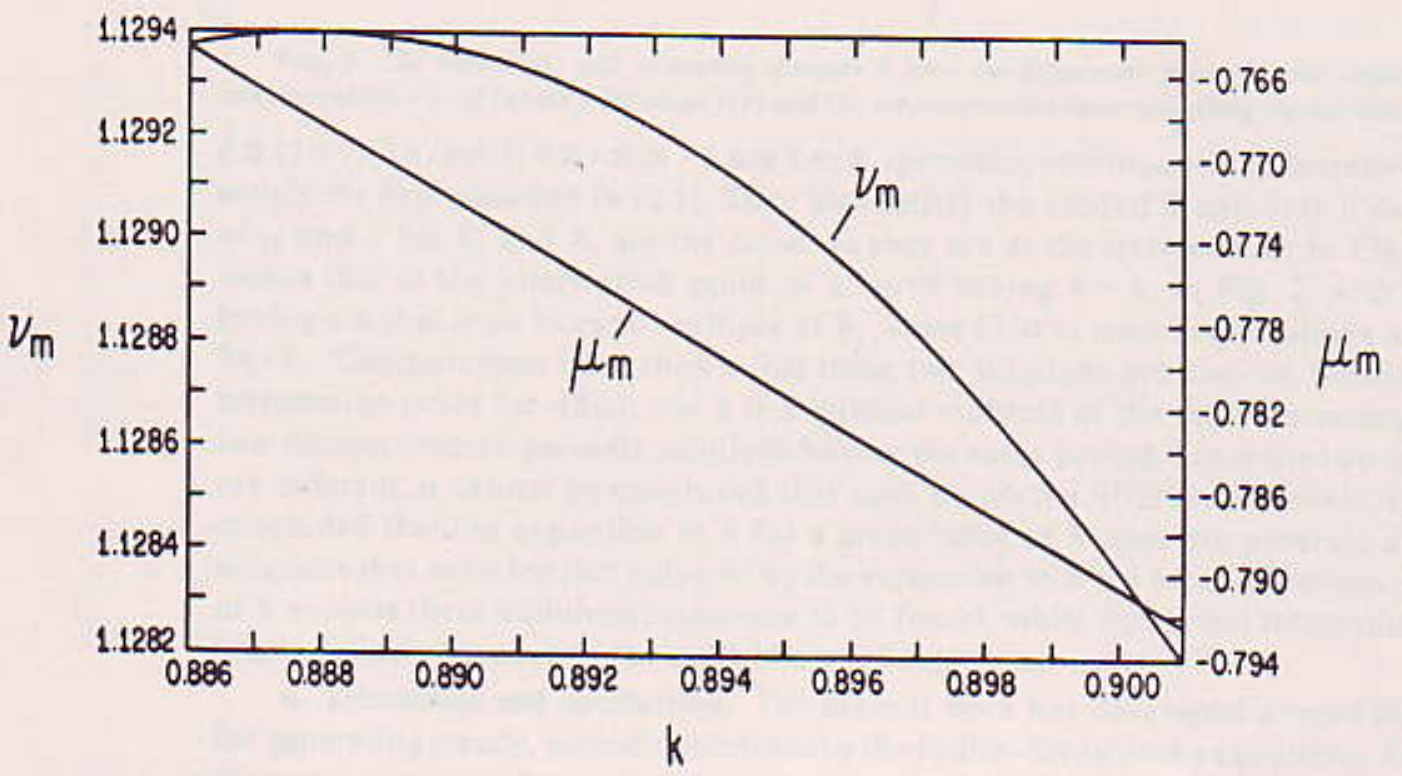

FIG. 4. The maximum value of $\nu$ and the value of $\mu$ at this maximum as functions of the wavenumber $k$ for the smooth perindic solutions. 

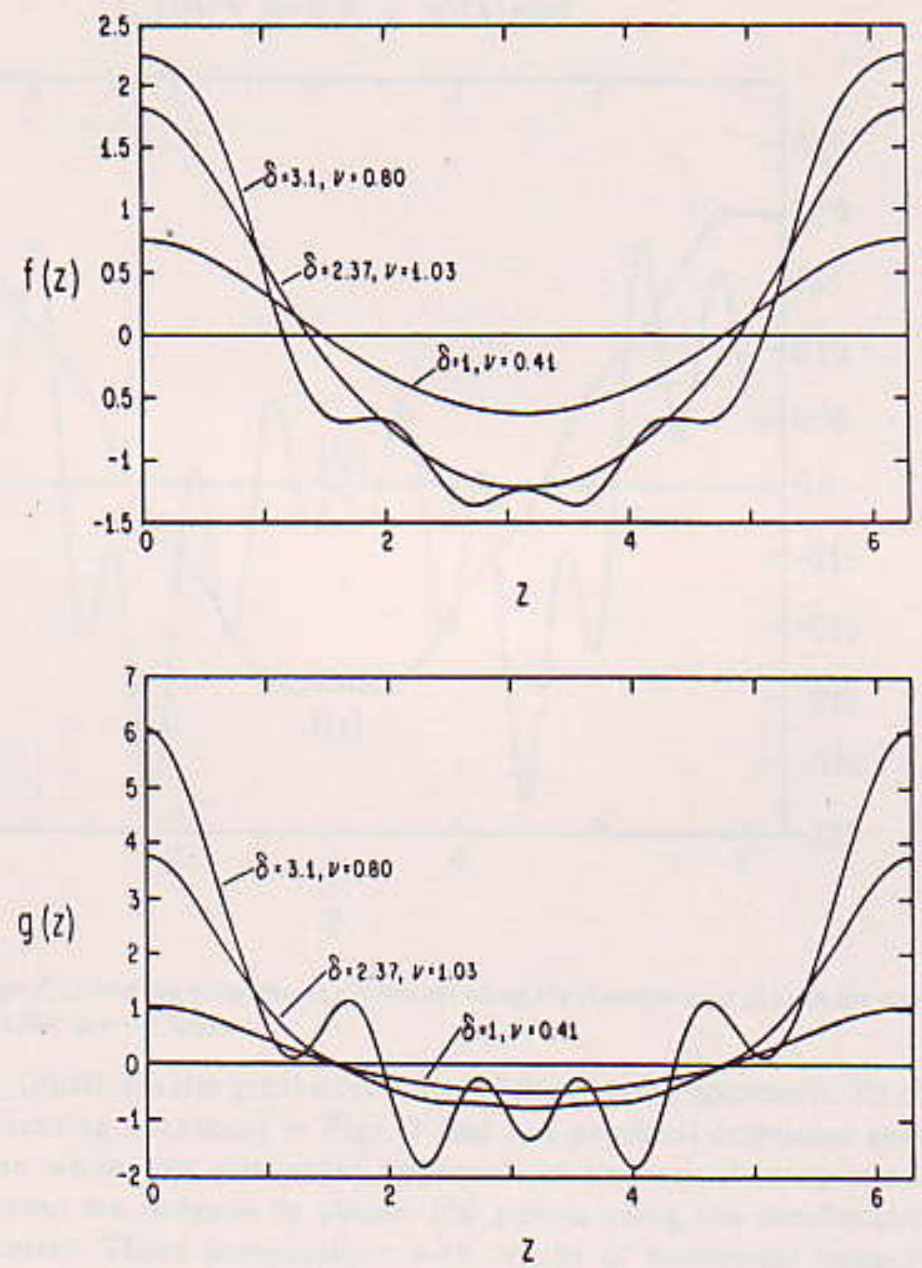

FIG. 5. The variations, with increasing distance $\delta$ from the bifurcation point, for the smooth periodic solutions with $k=1$, of (a) the front shape $f(z)$ and (b) the temperature decrement along the reaction zone $g(z)$.

$\zeta \leqq(j+1)\left(2 \pi / m k_{1}\right), 0 \leqq j \leqq m-1$ are $2 \pi / k_{1}$-periodic, continuously differentiable, and satisfy the first equation in (2.1). They also satisfy the second if and only if the values of $\mu$ and $\nu$ for $k_{1}$ and $k_{2}$ are the same, as they are at the intersections in Fig. 2. This means that at the intersection point of a curve having $k=k_{1}$ in Fig. 2, with another having a $k$ that is an integral multiple of $k_{1}$, there exist at least two solutions of period $2 \pi / k_{1}$. Computations have shown that these two solutions are distinct. Similarly, any intersection point for which one $k$ is a rational multiple of the other possesses at least two distinct smooth periodic solutions having the same period. Since the two solutions are different, it cannot be concluded that such points are bifurcation points. It can be concluded that the expansion in $\delta$ for a given value of $k$ does not generate all of the solutions that exist for that value of $k$; the expansion in $\delta$ for other appropriate values of $k$ enables these additional solutions to be found, while numerical integration could prove difficult because of the multiplicity of solutions.

6. Discussion and conclusions. The present work has developed a rapid algorithm for generating steady, periodic solutions to the Joulin-Sivashinsky equations. Although there are many methods of numerical integration for solving this particular system, comparison with the Richardson extrapolation method combined with a shooting 


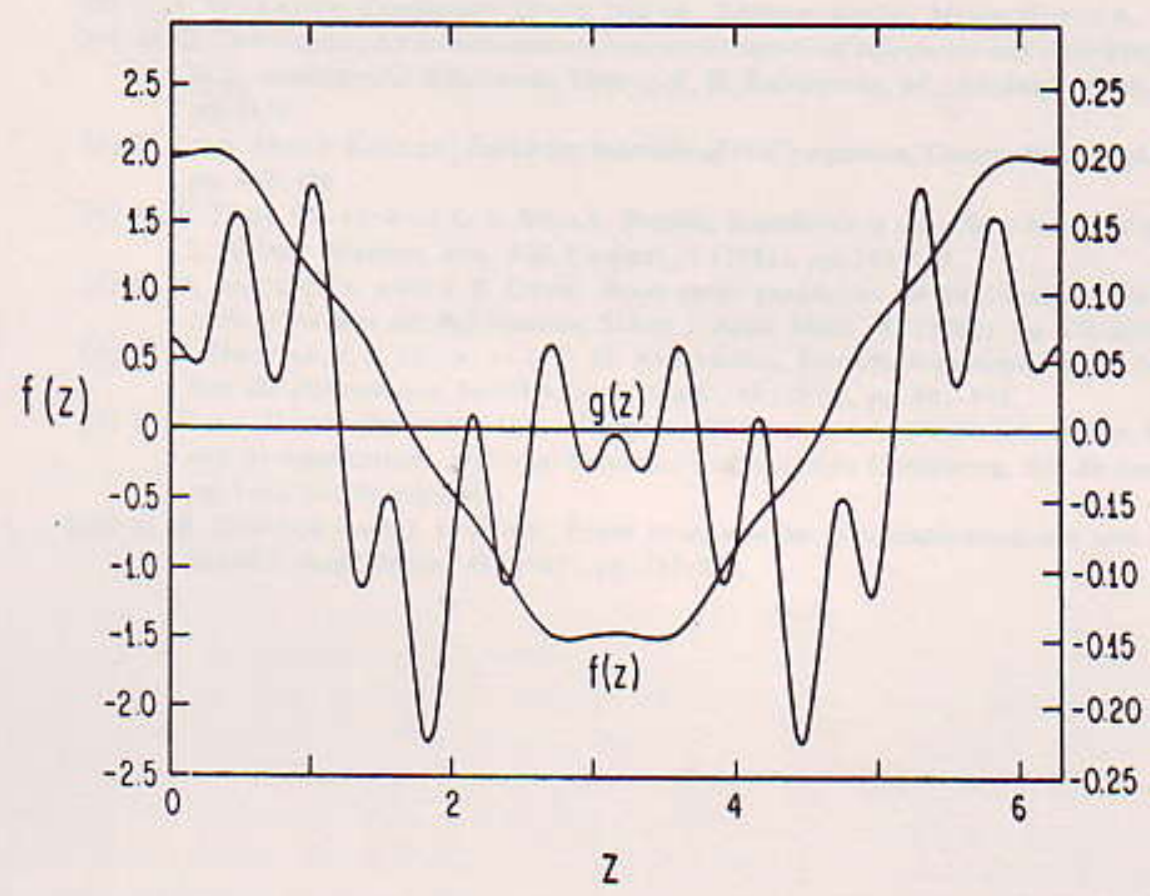

Fio, 6. The front shape $f(z)$ and the temperature decrement along the reaction zone $g(z)$ at the extremum in Fig. $4, k=0.888, \nu=1.1294, \mu=-0.7686$.

method, for example, illustrates the great advantage of the present approach. To obtain one point in the bifurcating parabolas in Figs. 2 and 3, a personal computer spent 14 seconds of CPU time when this particular approach to numerical integration was employed, while it spent six seconds to obtain 100 points using the combination of power and Fourier series. These comparisons with results of numerical integrations also have demonstrated very good accuracy for the algorithm. Many solutions have been found, and the existence of periodic solutions beyond the extinction point of the planar flame has been proven. However, much more remains to be done.

Stability analyses need to be performed for these solutions to ascertain which solutions may be observed in real flames. Solutions with excessive heat loss or with excessive flame-shape oscillations might be anticipated to be unstable. In the absence of stability studies, definitive conclusions concerning the physical reality of the solutions cannot be drawn. It is found that multiple solutions at a given value of $\nu$ are stable, then it may be concluded that more than one steady flame shape can be established experimentally by properly selecting initial conditions.

The present study has focused on planar, one-dimensional periodicities. It is also of interest to consider two-dimensional spatial operators in (1.1), to determine whether geometric effects may extend steady solutions to even larger values of $\nu$. The method employed herein can be readily extended to such an investigation, which would severely tax the capabilities of most methods based on direct numerical integrations.

\section{REFERENCES}

[1] G. JOULIN AND G. I. SIVASHINSKY, On the dynamics of nearly extinguished nanadiabatic cellular flames, Combust. Sci. Tech., 31 (1983), pp. 75-90.

[2] G. Joulin, Shapes, velocities and propagation limits of a nonadiabatic cellular Jame, Combust. Sci. Tech., 47 (1986), pp. 69-79. 
[3] F. A. WILl.aims, Combustion Theory, 2nd ed., Addison-Wesley, Menlo Park, CA, 1985, pp. 275, 351.

[4] M. G. CrANDALL, An introduction to consiructive aspects of bifurcation and the implicit funtcrion theorem, in Applications of Bifurcation Theory, P. H. Rabinowitz, ed., Academic Press, New York, 1977, pp. $1-35$. [S] D. LeVy AND J. KeLLeR, Instability intervals of Hill's equation, Comm. Pure Appl. Math., 16 (1963).
pp. $469-476$.

[6] M. C. F. DE SINAY AND L. R. SINAY, Stability boundaries in the elliptic restricted three-body problem, I. Periodic solutions, Mat. Apl. Comput., 1 (1982), pp. 143-152.

[7] C. M. ANDERSEN AND J. F. GEER. Power series expansions for the frequency and period of the limil cycle of the Van der Pol equation, SIAM J. Appl. Math., 42 (1982), pp. 678-693.

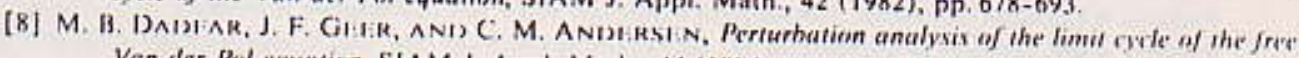
Van der Pol equation, SIAM J. Appl. Math., 44 (1984), pp. $881-895$.

[9] L. SiNAY, Iniroduction to bifurcarion theory, in VII School on Applied Mathematics, Bifurcation Theory and its Applications, National Laboratory of Scientific Computing, Rio de Janeiro, Brazil, 1986, pp. 1-63. (In Portuguese.)

[10] M. B. DADFAR AND J. F. GEF.R. Power series solution to a simple pendulum with oscillating support, SIAM J. Appl. Math., 47 (1987), pp. 737-750. 